ONLINE MUTATION REPORT

\title{
Clinical and molecular analysis of 30 patients with multiple lentigines LEOPARD syndrome
}

\author{
A Sarkozy, E Conti, M Cristina Digilio, B Marino, E Morini, G Pacileo, M Wilson, R Calabrò, \\ A Pizzuti, B Dallapiccola
}

J Med Genet 2004;41:e68 (http://www.jmedgenet.com/cgi/content/full/41/5/e68). doi: 10.1136/jmg.2003.013466

$M$ ultiple lentigines LEOPARD syndrome (MIM 151100) is an autosomal dominant multiple congenital anomaly syndrome, with high penetrance and markedly variable expression. ${ }^{1}$ The acronym LEOPARD was coined by Gorlin et al. in 1971 as a mnemonic of the major features of this disorder: multiple lentigines, ECG conduction abnormalities, ocular hypertelorism, pulmonic stenosis, abnormal genitalia, retardation of growth, and sensorineural deafness. ${ }^{2}$ It is also known as cardiocutaneous syndrome, Moynahan syndrome, lentiginosis profuse, and progressive cardiomyopathic lentiginosis. ${ }^{3}{ }^{4}$ Voron et al. proposed some diagnostic criteria for multiple lentigines LEOPARD syndrome. ${ }^{5}$ More than 100 cases have been described, and one review has been published. $^{56}$

Multiple lentigines LEOPARD syndrome shares many features with Noonan syndrome (MIM 163950), ${ }^{7-9}$ in which lentigines and deafness usually are not present. About $40 \%$ of patients with Noonan syndrome have missense mutations in the PTPN11 gene, which encodes for the protein tyrosine phosphatase SHP2. ${ }^{10-14}$ Multiple lentigines LEOPARD syndrome has proved to be allelic to Noonan syndrome, ${ }^{15}{ }^{16}$ with two recurrent PTPN11 mutations in exons 7 (Tyr279Cys) and 12 (Thr468Met). Recently, we reported a novel PTPN11 mutation (Gln506Pro) in a unique patient with multiple lentigines LEOPARD syndrome, which suggests that mutations other than Tyr279Cys and Thr468Met could be found in these patients. ${ }^{17}$ As PTPN11 mutations in multiple lentigines LEOPARD syndrome and Noonan syndrome are exclusive to these conditions, the distinctive manifestations of these disorders likely result from different molecular mechanisms. For instance, as commented in a recent report, the cardiac phenotypes in patients with Noonan syndrome and those with multiple lentigines LEOPARD syndrome with PTPN11 mutations are rather dissimilar, with pulmonary valve stenosis prevailing in the former and hypertrophic cardiomyopathy in the latter. ${ }^{18}$

We examined the PTPNII gene in a consecutive series of 30 patients with multiple lentigines LEOPARD syndrome, including 16 new patients. We report the full spectrum of molecular defects of these patients and describe the clinical features related to PTPN11 gene mutations.

\section{MATERIALS AND METHODS}

\section{Patient selection}

A clinical geneticist or a paediatric cardiologist chose the patients. Thirty patients of Caucasian origin ( 24 from central and southern Italy and six from Sydney, Australia), including 12 men and 18 women, were enrolled in this study. The age range was 8 months to 49 years (mean 16.37 (SD) 13.60 years). Clinical assessment for the diagnosis of multiple lentigines LEOPARD syndrome included physical evaluation of dysmorphisms and malformations, according to the clinical features outlined by Voron et al. ${ }^{5}$ Additional investigations included anthropometric measurements, renal ultrasonography,

\section{Key points}

- The clinical features and full spectrum of PTPN1 1 gene mutations in a large cohort of patients with multiple lentigines LEOPARD syndrome were investigated.

- Patients with multiple lentigines LEOPARD syndrome underwent clinical assessment that included evaluation of dysmorphisms and malformations, mostly according to the features outlined by Voron et al. Twenty-seven patients with full blown multiple lentigines LEOPARD syndrome were investigated. As a few signs of multiple lentigines LEOPARD syndrome may be age dependent, the study also included three unrelated children of unaffected parents, who did not have multiple lentigines but showed at least two cardinal features of multiple lentigines LEOPARD syndrome (such as hypertrophic cardiomyopathy and deafness).

- Of 24 index patients, 21 (88\%) harboured PTPN11 gene mutations. In addition to the three mutations reported previously, four novel mutations were identified: all were in exons 7, 12, and 13. Mutations were also found in patients who did not fulfil Voron's criteria.

- The clinical spectrum associated with PTPN11 mutations was markedly variable, with no pathognomonic feature. Common clinical features include multiple lentigines (86\%), facial dysmorphisms $(90 \%)$, congenital heart defects $(71 \%: 80 \%$ of these were hypertrophic cardiomyopathy), and sensorineural deafness (25\%). No obvious clinical difference was seen between patients with different mutations, except for bilateral deafness.

- Hypertrophic cardiomyopathy, the most prevalent heart defect in patients with mutations, should be searched for in otherwise asymptomatic individuals. Screening for PTPN1 1 mutations is recommended in patients who do not fulfil Voron's diagnostic criteria for multiple lentigines LEOPARD syndrome.

and radiological studies. In addition to multiple lentigines, other cutaneous abnormalities were recorded, particularly café au lait spots, axillary freckling, localised hypopigmentation, and other pigmented lesions. Patients with characteristic cephalofacial dysmorphisms, such as triangular face with biparietal bossing, hypertelorism, ptosis, epicanthic folds, prognathism, and large and low set auricles, were classified as mild or full dysmorphic. Cardiac evaluation included electrocardiogram, two dimensional echocardiography, and colour Doppler echocardiography. Pulmonary valve stenosis and pulmonary valve leaflet 
dysplasia were assessed according to classic echocardiographic criteria. Hypertrophic cardiomyopathy was diagnosed when the thickness of the left ventricular wall was more than two standard deviations from that of an age matched control group. ${ }^{19}$ Cardiac catheterisation with angiocardiography was performed in eight patients to attempt pulmonary valve dilatation or show the left ventricular shape and confirm the diagnosis. As a few signs of multiple lentigines LEOPARD syndrome are age dependent, we also included three unrelated children of nonaffected parents (aged eight months to four years), who were considered likely examples of multiple lentigines LEOPARD syndrome on the basis of café au lait spots, distinct facial characteristics, and congenital heart defects (two children) or hypertrophic cardiomyopathy and deafness without cutaneous abnormalities (one child).

The patients' parents, when available, were examined for major or minor dysmorphisms and cardiac and extracardiac defects. Informed consent was obtained from all patients or their parents. Cytogenetic analysis was normal in all patients.

\section{Molecular analysis}

We extracted DNA from the peripheral blood lymphocytes of all patients and available affected relatives. We screened the entire PTPN11 gene coding region, including the 15 exons and exon-intron boundaries (GenBank accession number NM_002834), for mutations. All fragments were amplified by polymerase chain reaction (PCR) with primers located about $50 \mathrm{bp}$ upstream and downstream from the splicing sites. We analysed the PCR fragments with single strand conformation polymorphism (Amersham Pharmacia Biotech, Uppsala, Sweden). We performed bidirectional direct sequencing on fragments with anomalous mobility shifts by using the Big Dye Terminator Abi Prism Sequencing Kit (Applied Biosystems, Foster City, CA) and the ABI PRISM 3100 Genetic Analyser Automated Sequencer (Applied Biosystems, Foster City, CA). We sequenced the entire PTPN11 coding region in samples with no anomalous mobility shifting fragments on single strand conformation polymorphism analysis.

\section{Statistical analysis}

Statistical analysis was performed with Fisher's exact test, with a significance threshold of $p \leqslant 0.05$.

\section{RESULTS}

The study group comprised 30 participants: 20 sporadic patients and 10 familial cases from four kindreds. We reevaluated 14 previously described participants and added 16 new patients, including the second sibling of a previously reported mother-sibling pair with multiple lentigines LEOPARD syndrome (patient N.15 (family B)) (table 1)..$^{15} 17$ Table 1 summarises the clinical findings. Twenty-seven patients fulfilled Voron's diagnostic criteria (patients N.124 and N.28-30), ${ }^{5}$ while three young patients were considered to have "partial" phenotypes of multiple lentigines LEOPARD syndrome because of a negative family history and absence of multiple lentigines in the presence of two or more other cardinal features of multiple lentigines LEOPARD syndrome (patients N.25-27). In particular, patient N.25 had severe hypertrophic cardiomyopathy, bilateral sensorineural deafness, hypotonia, and retarded developmental milestones but no cutaneous anomalies or facial dysmorphisms and patient N.26 had full facial dysmorphisms, hypertrophic cardiomyopathy, and three café au lait spots. Patient N.27 had hypertrophic cardiomyopathy with subaortic stenosis, a broad chest, 15 sparse café au lait spots, and facial anomalies, including epicanthal folds, bilateral palpebral ptosis, and dysmorphic ears.
The most common features among the 30 patients were multiple lentigines ( 25 patients), mild to full facial dysmorphisms (29 patients), and structural heart defects (18 patients; most often hypertrophic cardiomyopathy (14 patients)). Sensorineural deafness was diagnosed in six patients.

Analysis with single strand conformation polymorphism showed anomalous mobility shifting fragments in exons 7 , 12 , and 13 only. Of 24 index patients, 21 (88\%) harboured a PTPN11 gene mutation. The PTPN11 gene was mutated in $17 / 20$ sporadic patients and in all familial cases (table 1 ); the mutations cosegregated with the disease. Mutations were not found in the unaffected parents or in 200 control chromosomes. None of the mutations has been described previously in Noonan syndrome, with the notable exception of the Tyr279Cys mutation, which has been reported in a single patient with Noonan syndrome. ${ }^{11}$ We identified seven different missense PTPNII mutations, which clustered in the SHP2 protein tyrosine phosphatase domain (fig 1). Sixteen unrelated patients had one of the two common mutations ${ }^{15}$ : eight of these had the $836 \mathrm{~A} \rightarrow \mathrm{G}$ transition, which leads to a Tyr279Cys change and eight had the 1403C $\rightarrow$ T transition (Thr468Met). Seven patients had five rare mutations. One of these (Tyr279Ser in patient N. 25) occurred at nucleotide $836(836 \mathrm{~A}>\mathrm{C})$, which is also involved in the more common Tyr279Cys mutation. A G $>C$ transition at position 1391 in exon 12, which led to a Gly464Ala substitution, was found in a mother and her daughter (patients N.19 and N.20). Three other mutations were detected in exon 13. The Gln506Pro $(1517 \mathrm{~A}>\mathrm{C})$ change was reported in one sporadic patient (patient N.24). ${ }^{17}$ The remaining two sequence variations at codon 498 predicted different amino acidic substitutions. The first change was a $\mathrm{C}>\mathrm{T}$ transition at position 1492, which led to an Arg498Leu substitution (patient N.23); the second change was a G>T transversion at position 1493, which segregated from an affected mother to her daughter and predicted an Arg498Trp change (patients N.21 and N.22).

Three patients who satisfied Voron's criteria for multiple lentigines LEOPARD syndrome had no anomalous mobility pattern on single strand conformation polymorphism analysis (patients N.28-30), and direct sequencing of the whole PTPN11 coding region failed to show any change in nucleotides. Among patients with a negative molecular analysis, patient N.28 had multiple café au lait spots, axillary and neck freckling, facial cheratosis pylaris, valvular pulmonary stenosis, left sensorineural deafness, macrocephaly, and facial anomalies including prominent eyes with bilateral palpebral ptosis and dysmorphic ears. Patient N.29 had multiple lentigines, mitral valve dysplasia, broad chest, moderate mental retardation, macrocephaly, and facial anomalies including bilateral palpebral ptosis, hypertelorism, epicanthal folds, and dysmorphic ears. Patient N.30 had multiple lentigines, short stature, hypertrophic cardiomyopathy, broad chest with pectus carinatum, facial anomalies including prominent eyes, bilateral palpebral ptosis, and dysmorphic ears.

\section{DISCUSSION}

In this study, we analysed the PTPN11 gene in 30 patients with multiple lentigines LEOPARD syndrome. In addition to the two already described recurrent missense mutations (Tyr279Cys and Thr468Met) and the Gln506Pro mutation found in a single patient, four additional novel and rare changes were detected in the PTPN11 gene. Mutations were found in $88 \%$ of the index patients, which indicated that PTPNIl is the major gene behind multiple lentigines LEOPARD syndrome. Patients with multiple lentigines LEOPARD syndrome with PTPN11 mutations had marked 


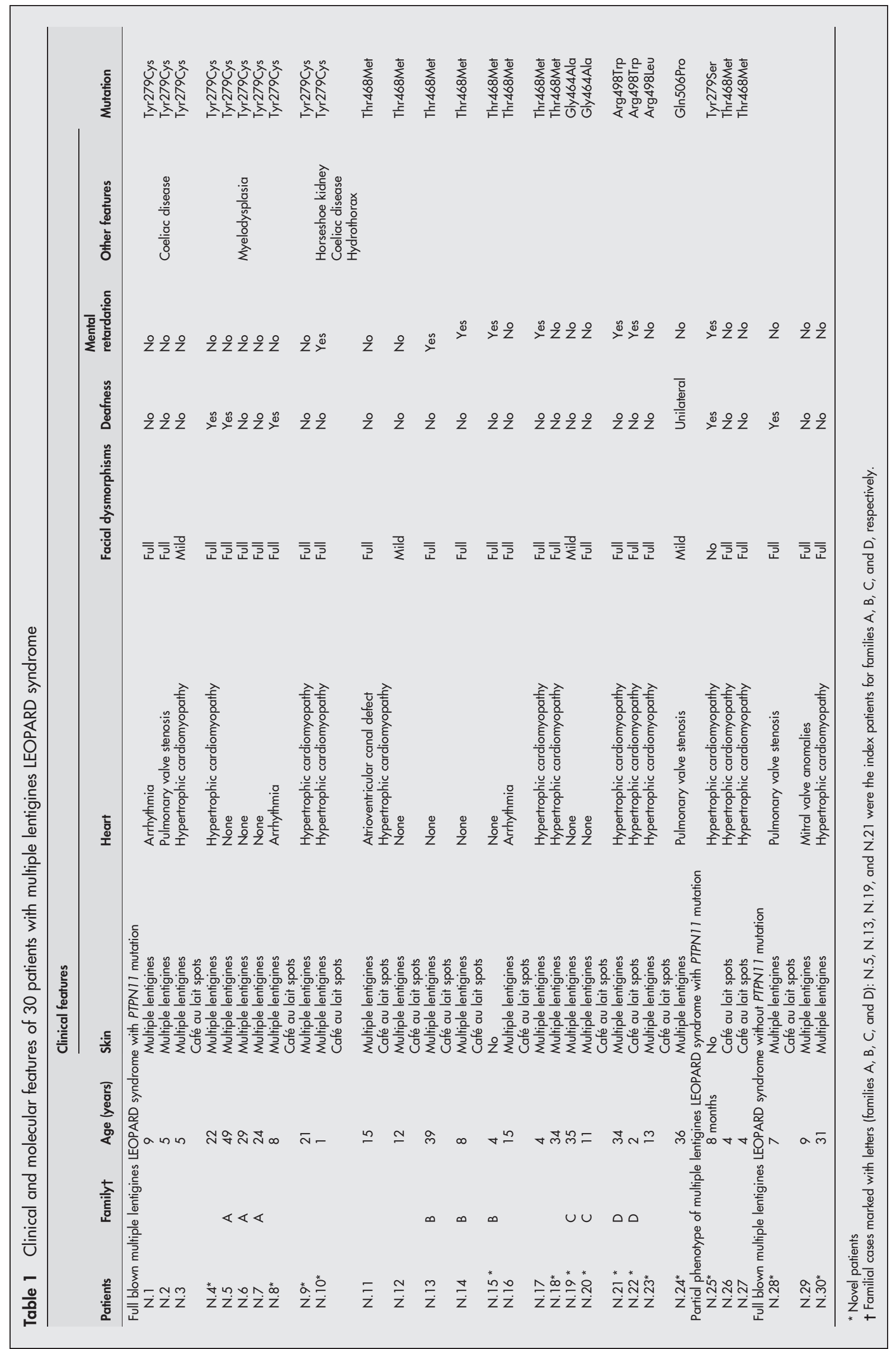




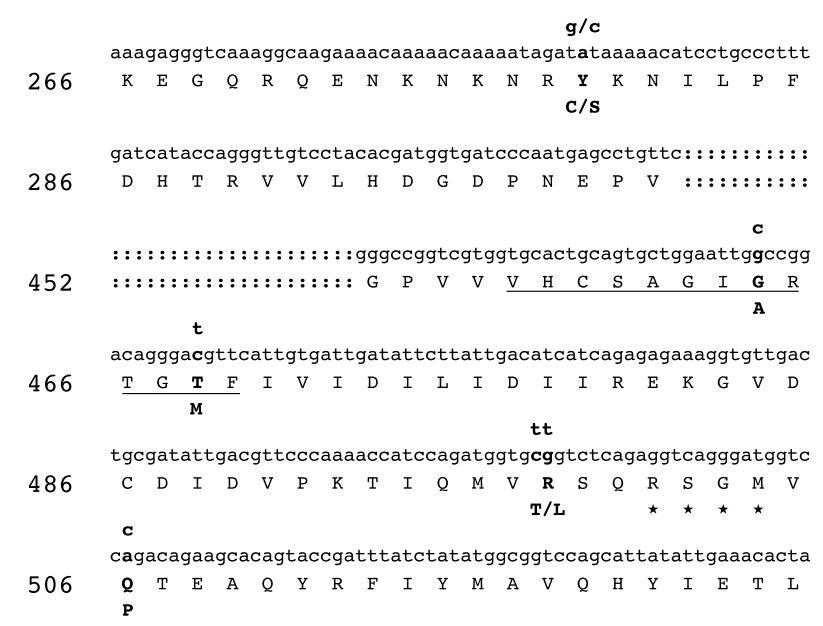

Figure 1 Nucleotide and amino acid sequence of the SHP2-PTP domain. Consecutive numbers indicates amino acid positions. Phosphatase active domain is underlined. Sequence variations and amino acidic changes in patients with multiple lentigines and Leopard syndrome are indicated above and below the wild type sequence.

* indicates positions of Noonan syndrome mutations located in exon 13.

variations in the clinical expression, which did not fulfil Voron's diagnostic criteria in about $14 \%$ of the cases.

All of the mutations occurred in exons that code for the protein tyrosine phosphatase domain (fig 1). The most common changes, in exons 7 (Tyr279Cys) and 12 (Thr468Met), accounted for $8 / 21$ (67\%) of mutations. One rare mutation involved the same nucleotide as the common Tyr279Cys change, but it produced a different amino acidic substitution (Tyr279Ser). Another rare mutation in exon 12 affected amino acid Gly464, which, like the more commonly mutated Thr468, is located in the consensus sequence of the active site that is necessary for activity of protein tyrosine phosphatase (amino acids 457-469, VHCSAGIGRTGTF). Two novel mutations were identified in exon 13. They both involved Arg498, which is located between the Thr468 mutation hot spot (Thr468Met) and Gln506, which was mutated in a sporadic patient with full blown multiple lentigines (Gln506Pro). ${ }^{17}$ These amino acids are in close proximity in the protein tyrosine phosphatase domain region, and they flank a mutation hot spot for Noonan syndrome, at amino acids 501-504. This region faces the N-terminal SH2 $(\mathrm{N}-\mathrm{SH} 2)$ domain, a mutation hot spot in patients with Noonan syndrome that has not been shown yet to be involved in multiple lentigines LEOPARD syndrome. On the other hand, mutations at position 464, 468, 498, and 506 have not been detected yet in patients with Noonan syndrome. All the amino acids mutated in patients with multiple lentigines LEOPARD syndrome are conserved in several related tyrosine phosphatases, which suggests a crucial role in the catalytic activity of the protein tyrosine phosphatase domain. Structural analysis of two of the mutations indicates a possible gain in phosphatase activity. ${ }^{15}$ Thr468, which is located in the active site of the consensus sequence, is a stabilising amino acid. Its substitution might alter the intrinsic structural equilibrium of the catalytic centre by altering the protein hydrogen bonding networks. ${ }^{15}$ Gln506, like other residues located in the N-SH2 and protein tyrosine phosphatase domains, is also involved in the same hydrogen bonding network. ${ }^{17}$ Gln506Pro substitution likely causes loss of the inhibitory effect of the N-SH2 domain on the phosphatase active site. Substitutions of the amino acid Gly464 in the active site consensus sequence and Thr498 in the active site of the protein tyrosine phosphatase domain might result in similar structural changes in the catalytic centre.

The rate of detection of PTPNII mutations in patients with multiple lentigines LEOPARD syndrome consistently is higher than in patients with Noonan syndrome. ${ }^{10-14}$ Although genetic heterogeneity has been established in patients with Noonan syndrome, ${ }^{10-14} 20$ it has not been proven in those with multiple lentigines LEOPARD syndrome. ${ }^{21}$ Although intronic and regulatory regions of the PTPN11 gene were not analysed in our study, our inability to detect mutations in the three unrelated children of unaffected parents could point to the supposed genetic heterogeneity of multiple lentigines LEOPARD syndrome.

This study investigated one of the largest cohorts of patients with multiple lentigines LEOPARD syndrome. In addition to 18 patients with mutations and a full blown phenotype of multiple lentigines LEOPARD syndrome, three patients with a partial phenotype of multiple lentigines LEOPARD syndrome showed PTPNII mutations in the two common mutation hot spots.

Distinct facial dysmorphisms, including hypertelorism, ptosis, and large low set auricles, occurred in $90 \%$ of patients with PTPNI1 mutations independently of age. As expected, multiple lentigines were present in most patients (86\%). They presented as flat, black-brown macules anywhere on the skin, but mostly on the face, neck, and upper part of the trunk. The number of lentigines increased into the thousands until puberty. Café au lait spots also were observed, alone or in association with multiple lentigines (61\%). Two children (patient N.15 (family B) and patient N.22 (family D)) with PTPN11 mutations, who were evaluated at the ages of four and two years, had no pigmented macules. Both children fulfilled Voron's criteria, however, because their mothers were affected (patients N.13 (family B) and N.21 (family D)) and onset of lentigines in the mothers occurred only in late childhood. Structural cardiac defects occurred in $71 \%$ of the index patients with PTPN11 mutations. Hypertrophic cardiomyopathy was the most common defect (12/15 (80\%) patients) and was not related to any specific mutation site. Pulmonary valve stenosis with valve leaflet dysplasia was less common than previously thought, affecting only $2 / 21$ patients with PTPN11 mutations. ${ }^{56915-17}$ Partial atrioventricular canal defect was diagnosed in one patient (patient N.11), who had developed hypertrophic cardiomyopathy during the past year, soon after the onset of multiple lentigines. In agreement with a previous report, ${ }^{22}$ we found that the first diagnosis or worsening of hypertrophic cardiomyopathy, or both, was concomitant with the appearance of multiple lentigines. This observation leads to the recommendation that any patient with isolated multiple lentigines or multiple lentigines LEOPARD syndrome should undergo careful cardiac assessment during infancy and adolescence. Hearing loss was documented at any age in about one quarter of patients. ${ }^{59}$ Interestingly, the four patients with bilateral sensorineural deafness who we evaluated harboured mutations at position 279, while a patient with the rare Gln506Pro mutation presented unilateral deafness. Coeliac disease was diagnosed in two (9.5\%) patients with mutations, a value significantly higher than that seen in the general population $(0.54 \%)(p=0.02)$ and comparable with that seen in patients with Down syndrome, Turner syndrome, and Williams syndrome. ${ }^{23-25}$ This observation deserves further evaluation in other patients to assess if coeliac disease must be included among the features of multiple lentigines LEOPARD syndrome, and screening for antigliadin and antiendomisium antibodies is indicated in these people. Mental retardation, if present, usually was mild, at times manifesting only with mild learning difficulties and a frequency comparable with that in previous 
studies, having been found in three sporadic patients and two families $(5 / 21,24 \%),{ }^{59}$ in association with different PTPN11 mutations.

Patients who presented with a "partial" phenotype of multiple lentigines LEOPARD syndrome and harboured PTPN11 mutations had an incomplete clinical spectrum, almost certainly because of their young age. Two patients (N.26 and N.27) showed the characteristic facial appearance, hypertrophic cardiomyopathy, and café au lait spots. The third patient (patient N.25) was aged eight months and had hypertrophic cardiomyopathy and congenital deafness, with no obvious facial dysmorphism or skin macules. The occurrence of hypertrophic cardiomyopathy with deafness is rare in young patients. In the absence of lentigines, Voron's criteria for the diagnosis of multiple lentigines LEOPARD syndrome include at least three major features and an immediate affected relative. ${ }^{5}$ Personal experience suggests that any young patient without multiple lentigines and a positive family history should be evaluated for the presence of de novo PTPN11 gene mutations when other features of multiple lentigines LEOPARD syndrome are found, particularly hypertrophic cardiomyopathy or pulmonary valve stenosis (with leaflet dysplasia) in association with deafness. The identification in two of these patients of the recurrent Thr468Met mutation may anticipate a later onset of multiple lentigines. On the other hand, in the presence of the newly recognised Tyr279Ser mutation, we could only guess that the full blown phenotype of multiple lentigines LEOPARD syndrome would develop in the forthcoming years. The clinical follow up of this patient and of the unique patient with Noonan syndrome who also harboured the common Tyr279Cys mutation of multiple lentigines LEOPARD syndrome should prove the assumption that codon 279 mutations are pathognomonic of the phenotype of multiple lentigines LEOPARD syndrome. ${ }^{11}$

Skin features, congenital heart disease, and mental status mostly were concordant in our four families with multiple lentigines LEOPARD syndrome. In particular, after the diagnosis of hypertrophic cardiomyopathy in a patient aged two years, the reevaluation of the affected mother in family D, who was aged 34 years, disclosed the same congenital heart disease. Familial concordance could be an important clue for the assessment of the reproductive risk of affected parents. $^{22}$ In this respect, the $25 \%$ occurrence rate of congenital heart disease in familial cases is lower than expected, possibly because of the reduced fitness of severe congenital heart disease. A number of families without congenital heart disease have been reported, which suggests that multiple lentigines might be an autosomal dominant disorder distinct from LEOPARD syndrome. ${ }^{26}$ Others have considered both disorders as unique clinical conditions, although the term LEOPARD has been restricted at times to patients who present with visceral abnormalities. ${ }^{27}$ Detection of the same PTPN11 mutation in patients with multiple lentigines, those with full blown multiple lentigines LEOPARD syndrome, and those with the "partial" phenotype of multiple lentigines LEOPARD syndrome suggests that these different phenotypes mark the variable expression of a unique genetic condition.

Clinical overlap between multiple lentigines LEOPARD syndrome and neurofibromatosis 1 is well known. In fact, café au lait spots and multiple lentigines are skin manifestations common to both disorders. Café au lait spots increase in number with age in both conditions, whereas the lentigines characteristic of multiple lentigines LEOPARD syndrome are not present at younger ages and develop during childhood, so that differential diagnosis in children may be difficult. On the contrary, the lentigines of multiple lentigines LEOPARD syndrome in adolescence or adult age are distinguishable from those of neurofibromatosis 1, being generalised and dark in colour. In addition, in children with café au lait spots, the presence of facial anomalies or congenital heart disease may be more suggestive for multiple lentigines LEOPARD syndrome, although intermediate phenotypes such as neurofibromatosis 1-Noonan syndrome or Watson syndrome have been described. In practical genetic evaluation, we believe that PTPNIl screening should be carried out at first in children with café au lait spots associated with facial anomalies or congenital heart disease (hypertrophic cardiomyopathy or pulmonary valve stenosis), or both, although in the absence of multiple lentigines. In addition, PTPN11 analysis should be considered in patients with café au lait spots or multiple lentigines when neurofibromas or Lisch nodules are not detectable and molecular analysis for mutations of NFI is negative. Conversely, screening of the NFl gene should be performed in patients with skin manifestations of multiple lentigines LEOPARD syndrome without a detectable PTPNII mutation. Nevertheless, the existence of a third locus for multiple lentigines LEOPARD syndrome also should be considered.

In our series, no obvious clinical difference was seen between patients with allelic mutations, except for bilateral sensorineural deafness, which we found always in association with mutations of the amino acid Tyr279 (table 1) $(p=0.021)$, although one patient with monolateral deafness carried a mutation in exon 13. Screening of additional patients with multiple lentigines LEOPARD syndrome with bilateral and monolateral deafness is needed to confirm or exclude this observation. Clinical variability seen in patients with the same mutation likely reflects the effect of modifier genes or environmental factors, or both, which influences the phenotypes associated with PTPNII mutations.

The high prevalence of hypertrophic cardiomyopathy in patients with multiple lentigines LEOPARD syndrome and PTPNII mutations (57\%) indicates that mutations related to multiple lentigines LEOPARD syndrome confer an increased risk for this congenital heart disease. This conclusion also seems to be strengthened by the relatively rare occurrence of hypertrophic cardiomyopathy in Noonan syndrome (15\%), ${ }^{18}$ where, in general, PTPN11 mutations cluster to the N-SH2 domain. ${ }^{10-14} 18$ Detection of hypertrophic cardiomyopathy, therefore, should be considered an important hint in the search for PTPNIl mutations in the absence of multiple lentigines.

\section{Conclusion}

PTPN11 is the major gene for multiple lentigines LEOPARD syndrome, being mutated in about $90 \%$ of patients with the condition. Seven different mutations have been identified so far. Gene analysis in these patients should first include screening by direct sequencing for mutations in exons 7 and 12 , which are present in $86 \%$ of the cases, and then analysis of exon 13. The phenotypic spectrum of patients with mutations is associated with a marked clinical variation in the absence of any pathognomonic feature. Hypertrophic cardiomyopathy should be suspected and screened for in patients with multiple lentigines LEOPARD syndrome who do not harbour PTPN11 mutations, and careful cardiac evaluation and follow up also should be performed in asymptomatic people. Identification of PTPN11 mutations also in patients with the "partial" phenotype of multiple lentigines LEOPARD syndrome suggests the inclusion in the analysis of people who do not fulfil Voron's diagnostic criteria. Consequently, the diagnosis of multiple lentigines LEOPARD syndrome should not be ruled out in any young patients with no multiple lentigines and a negative family history who present with hypertrophic cardiomyopathy or 
pulmonary valve stenosis in association with an extracardiac feature, such as hearing loss.

\section{ACKNOWLEDGEMENTS}

We thank Dr Kristi Jones (Department of Clinical Genetics, Children's Hospital, Sydney) for choosing the patients and Dr Giorgia Esposito (CSS- Mendel Institute, Rome) for technical assistance.

\section{Authors' affiliations}

A Sarkozy, E Conti, E Morini, A Pizzuti, B Dallapiccola, CSS Hospital, IRCCS, San Giovanni Rotondo, Italy, and CSS- Mendel Institute, Rome, Italy

A Sarkozy, E Morini, A Pizzuti, B Dallapiccola, Department of Experimental Medicine and Pathology, University "La Sapienza", Rome, Italy

M C Digilio, Division of Medical Genetics, Bambino Gesù Hospital, IRCCS, Rome, Italy

B Marino, Section of Pediatric Cardiology, Institute of Pediatrics, University "La Sapienza", Rome, Italy

G Pacileo, R Calabrò, Pediatric Cardiology, Monaldi Hospital, Naples, Italy

M Wilson, Department of Clinical Genetics, The Children's Hospital, Sydney, Australia

Funding: This study was supported in part by grants from the Italian Ministry of Health (RC 2002 and 2003) and from the Italian Ministry of Instruction, University and Research (Grant 40\% to BD).

Conflicts of interest: none declared.

Correspondence to: Dr Pizzuti, Istituto CSS-Mendel, Viale Regina Margherita 261, 00198 Rome, Italy; a.pizzuti@css-mendel.it

Revised version received 25 August 2003

Accepted for publication 24 October 2003

\section{REFERENCES}

1 Gorlin RJ, Anderson RC, Blaw M. Multiple lentigines syndrome. Am J Dis Child 1969; 17:652-62

2 Gorlin RJ, Anderson RC, Moller JH. The leopard (multiple lentigines) syndrome revisited. Birth Defects Orig Artic Ser 1971;7:110-5.

3 Moynahan EJ. Progressive cardiomyopathic lentiginosis: first report of autopsy findings in a recently recognized inheritable disorder (autosomal dominant). Proc R Soc Med 1970;63:448-51.

4 Seuanez H, Mane-Garzon F, Kolski R. Cardio-cutaneous syndrome (the "LEOPARD" syndrome). Review of the literature and a new family. Clin Genet 1976;9:266-76.

5 Voron DA, Hatfield HH, Kalkhoff RK. Multiple lentigines syndrome. Case report and review of the literature. Am J Med 1976:60:447-56.

6 Gorlin JR, Cohen MM, Hennekam RCM, eds. Syndromes of the head and neck. New York: Oxford University Press, 2001:555-8.

7 Allanson JE. Noonan syndrome. J Med Genet 1987;24:9-13.

8 Blienden LC, Schneeweiss A, Shem-Tov A, Feigel A. Neufeld HN. Unifying link between Noonan's and Leopard syndromes? Pediatr Cardiol 1983:4:168-9.

9 Coppin BD, Temple IK. Multiple lentigines syndrome (LEOPARD syndrome or progressive cardiomyopathic lentiginosis). J Med Genet 1997;34:582-6.
10 Tartaglia M, Mehler EL, Goldberg R, Zampino G, Brunner HG, Kremer H, van der Burgt I, Crosby AH, lon A, Jeffery S, Kalidas K, Patton MA, Kucherlapati RS, Gelb BD. Mutations in PTPN1 1, encoding the protein tyrosine phosphatase SHP-2, cause Noonan syndrome. Nat Genet 2002;30:123-6.

11 Tartaglia M, Kalidas K, Shaw A, Song X, Musat DL, van der Burgt I, Brunner HG, Bertola DR, Crosby A, lon A, Kucherlapati RS, Jeffery S, Patton MA, Gelb BD. PTPN1 1 mutations in Noonan syndrome: molecular spectrum, genotype-phenotype correlation, and phenotypic heterogeneity. Am J Hum Genet 2002;70:1555-63.

12 Maheshwari M, Belmont J, Fernbach S, Ho T, Molinari L, Yakub I, Yu F, Combes A, Towbin J, Craigen WJ, Gibbs R. PTPN1 1 mutations in Noonan syndrome type I: detection of recurrent mutations in exons 3 and 13. Hum Mutat 2002:20:298-304.

13 Musante L, Kehl HG, Majewski F, Meinecke P, Schweiger S, GillessenKaesbach G, Wieczorek D, Hinkel GK, Tinschert S, Hoeltzenbein M, Ropers HH, Kalscheuer VM. Spectrum of mutations in PTPN1 1 and genotypephenotype correlation in 96 patients with Noonan syndrome and five patients with cardio-facio-cutaneous syndrome. Eur J Hum Genet 2003;11:201-6.

14 Kosaki K, Suzuki T, Muroya K, Hasegawa T, Sato S, Matsuo N, Kosaki R, Nagai T, Hasegawa Y, Ogata T. PTPN1 1 (protein-tyrosine phosphatase, nonreceptor-type 11) mutations in seven Japanese patients with Noonan syndrome. J Clin Endocrinol Metab 2002;87:3529-33.

15 Digilio MC, Conti E, Sarkozy A, Mingarelli R, Dottorini T, Marino B, Pizzuti A, Dallapiccola B. Grouping of multiple-lentigines/LEOPARD and Noonan syndromes on the PTPN11 gene. Am J Hum Genet 2002;71:389-94.

16 Legius E, Schrander-Stumpel C, Schollen E, Pulles-Heintzberger C, Gewillig M, Fryns JP. PTPN 11 mutations in LEOPARD syndrome. J Med Genet 2002;39:571-4

17 Conti E, Dottorini T, Sarkozy A, Tiller GE, Esposito G, Pizzuti A, Dallapiccola B. A novel PTPN1 1 mutation in LEOPARD syndrome. Hum Mut Mutation in Brief 2003;21:645.

18 Sarkozy A, Conti E, Seripa D, Digilio MC, Grifone N, Tandoi C, Fazio VM, Di Ciommo V, Marino B, Pizzuti A, Dallapiccola B. Correlation between PTPN1 1 gene mutations and congenital heart defects in Noonan and LEOPARD syndromes. J Med Genet 2003:40:704-8.

19 Burch M, Sharland M, Shinebourne E, Smith G, Patton M, McKenna W. Cardiologic abnormalities in Noonan syndrome: phenotypic diagnosis and echocardiographic assessment of 118 patients. J Am Coll Cardiol 1993;22: $1189-92$

20 Jamieson $C R$, van der Burgt I, Brady AF, van Reen M, Elsawi MM, Hol F, Jeffery S, Patton MA, Mariman E. Mapping a gene for Noonan syndrome to the long arm of chromosome 12. Nat Genet 1994;8:357-60.

21 Wu R, Legius E, Robberecht W, Dumoulin M, Cassiman JJ, Fryns JP. Neurofibromatosis type I gene mutation in a patients with features of LEOPARD syndrome. Hum Mutat 1996;8:51-6.

22 Somerville J, Bonham-Carter RE. The heart in lentiginosis. Br Heart J 1972;34:58-66.

23 Castro M, Crino A, Papadatou B, Purpura M, Giannotti A, Ferretti F, Colistro F, Mottola L, Digilio MC, Lucidi V. Down's syndrome and celiac disease: the prevalence of high IgA-antigliadin antibodies and HLA-DR and DQ antigens in trisomy 21. J Pediatr Gastroenterol Nutr 1993;16:265-8.

24 Ivarsson SA, Carlsson A, Bredberg A, Alm J, Aronsson S, Gustafsson J, Hagenas L, Hager A, Kristrom B, Marcus C, Moell C, Nilsson KO, Tuvemo T, Westphal $O$, Albertsson-Wikland K, Aman J. Prevalence of coeliac disease in Turner syndrome. Acta Paediatr 1999;88:933-6.

25 Giannotti A, Tiberio G, Castro M, Virgilii F, Colistro F, Ferretti F, Digilio MC, Gambarara M, Dallapiccola B. Coeliac disease in Williams syndrome. J Med Genet 2001;38:767-8.

26 Arnsmeier SL, Paller AS. Pigmentary anomalies in the multiple lentigines syndrome: Is it distinct from LEOPARD syndrome? Pediatr Dermatol 1996; 13:100-4.

27 Online Mendelian Inheritance in Man. A directory of human genes and genetic disorders. Bethesda, MD: National Centre for Biotechnology Information. http://www.ncbi.nlm.nih.gov/entrez/query.fcgi?db=OMIM 